\title{
Characterization of Gas Jet Behavior at a Submerged Orifice in Liquid Metal*
}

\author{
By Kazumi MORI,** Yasuhisa OZAWA*** and \\ Masamichi SANO**
}

\begin{abstract}
Synopsis
The present paper is concerned with a study made with the purpose of clarifying the behavior of injected gas jets especially at the exit of a submerged orifice in liquid metal. Nitrogen was injected into a mercury bath through an orifice of $0.1 \sim 0.4 \mathrm{~cm}$ in diameter located at the transparent vessel bottom. The gas-flow rates were varied from 0.05 to $4500 \mathrm{~cm}^{3} / \mathrm{s}$ at orifice conditions. The highest gauge pressure supplied to the system was $20 \mathrm{~kg} f / \mathrm{cm}^{2}$. Behavior of gas jets was observed directly through the bottom plate by using a high speed cinecamera. At low gas-flow rates the jet is shown to expand immediately upon discharging and form seemingly discrete bubbles. This behavior is called "bubbling". With increased gas-flow rates, an apparent coincidence between the base diameter and the orifice diameter begins to occur over various time ranges. In these time ranges the injected gas is considered to leave the orifice as a continuous jet of gas. This phenomenon is called " jetting" and is found to occur in the sonic flow region. The change from bubbling to jetting takes place in a transitional gas-flow range. With increasing gas-flow rate jetting fraction increases and bubbling fraction decreases.
\end{abstract}

\section{Introduction}

Submerged gas injection is a very important technique in modern steelmaking processes. Notable examples of processes relying on gas injection are oxygen-blow steelmaking processes (Q-BOP, AOD), new combined oxygen steelmaking processes, and gasstirring in ladle metallurgy. Although the importance of the behavior of submerged gas jets in gas injection processes has been very well recognized, our understanding of jet behavior is still meager. Better understanding of jet behavior will be useful in solving serious process problems such as refractory erosion due to jet action and clogging of tuyeres by frozen metal. In many of previous studies water was employed to model molten metals. Considering that physical properties of water are much different from those of molten metals, the authors have used mercury in a program of work on model-studies of gas injection in metallurgical systems. ${ }^{1-4)}$ The present paper is concerned with an investigation of the behavior of injected gas jets at a submerged orifice in mercury over a wide range of gas-flow rates extending to the region of sonic velocity.

In the previous studies the opacity of mercury has been a large obstacle in clarifying the behavior of gas jets. A visual technique was employed in the present study. Nitrogen was injected into a mercury bath through an orifice located at the transparent vessel bottom. Behavior of gas jets was photographed directly through the bottom plate by a high speed cinecamera. Additionally, the size of bubbles form- ed at the orifice was measured by sensing frequency of bubble formation.

\section{Experimental}

Figure 1 shows a schematic drawing of the experimental apparatus. The vessel containing mercury was a stainless steel cylinder of $10 \mathrm{~cm}$ in ID and $40 \mathrm{~cm}$ in height, the bottom of which was a transparent plate of 20 or $30 \mathrm{~mm}$ in thickness. An orifice was installed at the center of the bottom plate.

The gas lines consisted of a 3/8-inch copper pipe and a stainless steel pipe of $2 \mathrm{~mm}$ in ID or $4 \mathrm{~mm}$ in ID, the two pipes being connected by a rubber tube or a stainless steel joint. The stainless steel pipe was joined to the side of the vessel bottom plate. The rate of gas flow to the system was controlled by a pressure-regulating valve in series with a needle valve. The latter was not used in the case of gas blowing pressure higher than $8 \mathrm{kgf} / \mathrm{cm}^{2}$ in gauge.

The pressure in the vessel space above the liquid was measured to obtain the hydrostatic pressure at the bottom orifice. Downstream from the mercury vessel were located a gas reservoir of $1000 \mathrm{~cm}^{3}$ and a gas-flow meter. The gas reservoir was used so that the cyclical fluctuations of gas pressure produced from bubbling in the liquid did not disturb the measurement of gas-flow rate. Three types of gas-flow meter were used. For gas-flow rates lower than $30 \mathrm{~cm}^{3} / \mathrm{s}$ the flow rate was measured by a soap-film flow meter. From 30 to $1000 \mathrm{~cm}^{3} / \mathrm{s}$ a float meter was used. A differential pressure flow meter was used in the range

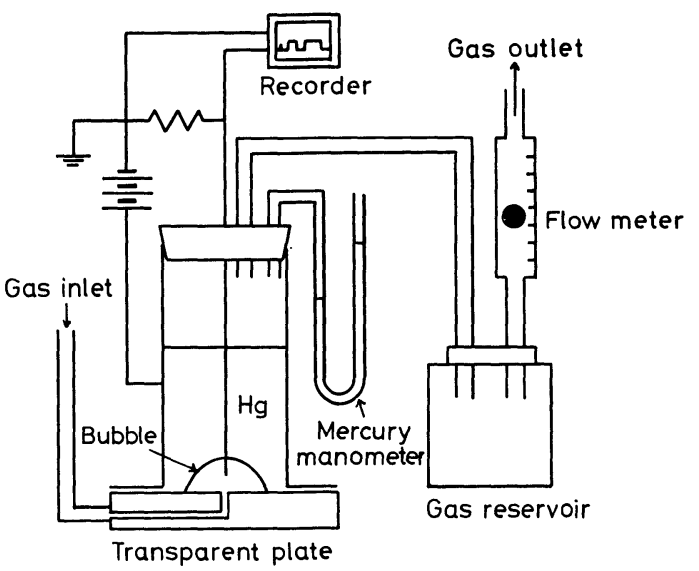

Fig. 1. Schematic drawing of experimental apparatus.

* Presented to the 96th ISIJ Meeting, October 1978, at Toyama University in Toyama. Manuscript received May 6, 1981.

** Department of Iron and Steel Engineering, Nagoya University, Chikusa-ku, Nagoya 464.

*** Graduate School of Engineering, Nagoya University. 
of gas-flow rates higher than $1000 \mathrm{~cm}^{3} / \mathrm{s}$.

A high speed cinecamera was positioned at $50 \mathrm{~cm}$ from the orifice. The film speed was 200 1 000 frames per second.

The bubble size was determined from the gas-flow rate and the frequency of bubble formation. The latter was measured by using an electro-resistivity probe. The tip of a molybdenum probe was of the size of $0.2 \mathrm{~mm}$ in diameter. The opening and closing of the circuit caused by the passing of bubbles over the probe tip produced square waves of frequency on an oscillograph.

At the beginning of each experiment, the probe was positioned on the predetermined level above the orifice. The level was selected at $6 \mathrm{~mm}$ above the orifice except for gas-flow rates lower than $30 \mathrm{~cm}^{3} / \mathrm{s}$, where the level was at $10 \mathrm{~mm} .{ }^{*}$ The vessel was then filled with mercury to a depth of $20 \mathrm{~cm}$. A high speed cinecamera was focused on the orifice. During gas injection, photographing of bubble formation and the measurement of its frequency were made simultaneously.

Experiments were made for orifices of $0.1,0.2,0.3$ and $0.4 \mathrm{~cm}$ in diameter. The gas-flow rates were varied from 0.05 to $4500 \mathrm{~cm}^{3} / \mathrm{s}$ at orifice conditions. Here, correction was made for the hydrostatic pressure (Eq. $\left.(\mathrm{A}-1)^{* *}\right)$. The highest pressure supplied to the system was $20 \mathrm{kgf} / \mathrm{cm}^{2}$ in gauge. All the experiments were made under the condition where the gas chamber volume did not produce any effect on jet behavior.

Figure 2(a) is a schematic drawing of a bubble forming from the orifice. Here, $\theta_{c}$ is the contact angle, $d_{o}$ is the orifice diameter, and $d_{i}$ is the instantaneous base-diameter of the bubble. Figure 2(b) shows an example of photograph of the bubble-base. A bubble, the base-diameter of which was as large as $3 \mathrm{~cm}$, was observed. Except for very low gas-flow rates, perfect circle of the bubble base was never observed. The base diameter of bubbles was therefore defined as the geometric mean of major and minor axes of an ellipse postulated for the base of a bubble.

\section{Bubbling Flow Regime}

In the present study, two flow regimes have been observed. At low gas-flow rates, the jet is shown to expand very rapidly immediately upon discharging from the orifice and form seemingly discrete bubbles.*** This phenomenon is called "bubbling". At gas-flow rates higher than a critical rate, another phenomenon called "jetting" occurs in a manner in which periods of jetting alternate with periods of bubbling. Description on jetting will be presented in the next chapter.

\section{Observation of the Base of Bubbles}

At very low rates of gas-flow the formation of bubbles is very regular. At higher flow rates the forma- tion becomes increasingly irregular.

Figure 3 is an example of the observation of the base of bubbles representing how the base diameter $\left(d_{i}\right)$ of bubbles changes with time. Each wave corresponds to one bubble. A maximum value, $d_{M}$, is defined for each bubble as the maximum base diameter of the bubble.

To handle the voluminous data by an electronic computer, a simplified representation was made as shown in Fig. 3 by rectangular waves. Here, the formation of a bubble is represented by a rectangular wave the height of which is equal to the base diameter $\left(d_{M}\right)$.

Figure 4 is an example of rectangular waves showing successive formation of bubbles. With increasing gas-flow rates for an orifice, bubble formation becomes increasingly irregular. However, a general trend of increase of the base diameter is observed.

To depict features of bubble formation of a given condition of gas injection, distribution function of base diameter $\left(d_{M}\right)$ is introduced. The cumulative distribution function, $F\left(d_{M}\right)$, is defined as the ratio of the

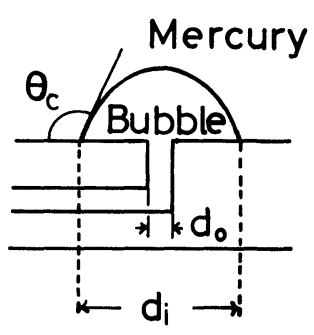

(a)

(a) Schematic drawing

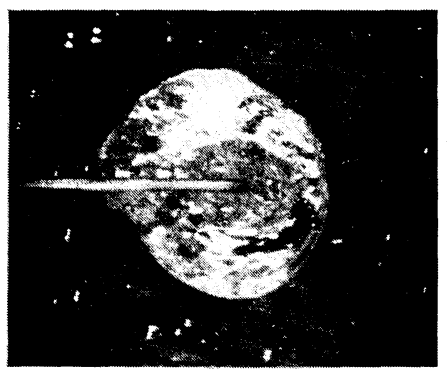

(b) (b) High-speed photograph $\left(d_{o}=0.2 \mathrm{~cm}, V_{g}=570 \mathrm{~cm}^{3} / \mathrm{s}\right)$ $(\times 1.0)(\times 3 / 4)$

Fig. 2. Bubble formation at the orifice.

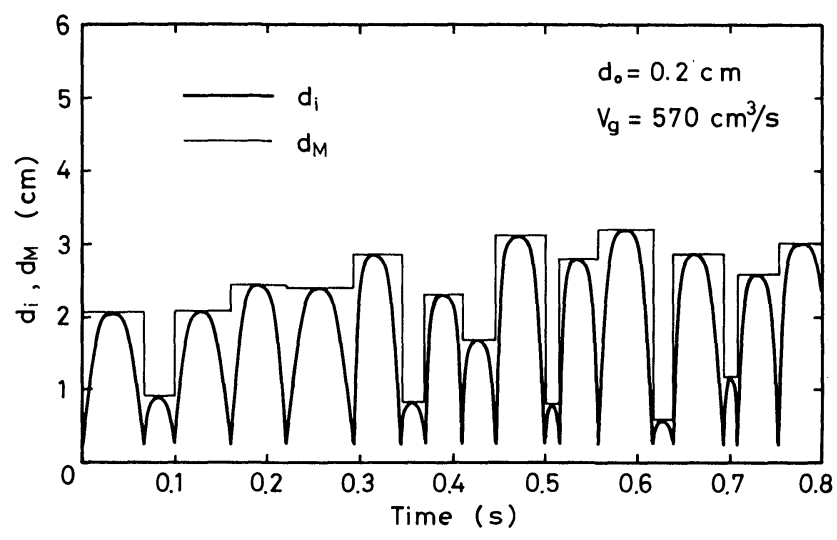

$d_{i}:$ instantaneous base diameter $d_{M}:$ maximum base diameter

Fig. 3. Time change of base diameter of bubbles forming at the orifice.

* The levels were selected after careful examination of a previous study. ${ }^{4}$ )

** The derivation of equations relating to gas-flow through the submerged orifice is given in Appendix.

*** The experiment of bubble size measurement has verified the formation of bubbles in the present case of mercury. 
sum of formation times of bubbles whose base diameters are equal to or smaller than $d_{M}$ to the total formation time of all bubbles in a photographing.* Figure 5 is an example of the distribution function. Here, for the sake of simplicity, the original step-wise representation is approximated by a curve.

From comparing the curves of distribution function for various orifice diameters at a given gas-flow rate, it is noticed that although the shape of each of the curve is much dependent upon the orifice diameter, the largest base diameter, denoted by $d_{M L}$, for each injecting condition is almost the same.

A bubble of base diameter $d_{M L}$ is considered to be least influenced by liquid circulation during its formation. The relation between the largest base diameter of bubbles and gas-flow rates is plotted in Fig. 6.** The gas-flow rates were varied as large as $10^{5}$ times. As seen from the figure, the most significant factor controlling the size of bubble base is gas-flow rate, orifice diameter exerting only minor effect.

At gas-flow rates $\left(V_{g}\right)$ lower than $2 \sim 3 \mathrm{~cm}^{3} / \mathrm{s}, d_{M L}$ is constant independently of both gas-flow rate and orifice diameter. This suggests that bubble formation is made under the static condition. At higher gas-flow rates $d_{M L}$ increases with increasing gas-flow rate. At $V_{g}>40 \mathrm{~cm}^{3} / \mathrm{s}$, the value of $d_{M L}$ depends a little on the diameter of the orifice.

\section{Calculations of the Base of Bubbles}

A calculation was made on the static formation of bubbles by using a theory developed by Fritz ${ }^{5)}$ for boiling. According to this theory, there is a maximum base diameter of bubble for a given contact angle. Giving $13.6 \mathrm{~g} / \mathrm{cm}^{3}$ and 480 dyne $/ \mathrm{cm}$ for the density and surface tension of mercury, respectively, one obtains maximum base diameters for the experimental value of contact angle ${ }^{6)}$ as shown in Fig. 6. Agreement between experiment and calculation seems to be reasonable. However, since the bubble diameter changes, if slightly, with the gas-flow rate, ${ }^{* * *}$ bubbles are formed not exactly under the static condition.

It is presumed that at $V_{g}<0.1 \mathrm{~cm}^{3} / \mathrm{s}$ static bubble formation occurs. $* *$ Details of mechanism of bubble formation at $0.1<V_{g}<2 \sim 3 \mathrm{~cm}^{3} / \mathrm{s}$ is not clear.

The results at higher gas-flow rates may be explained by the point source model developed by Davidson and Schüler ${ }^{7)}$ for bubble formation at a submerged orifice in a liquid. A spherical bubble the lower part of which is cut by a horizontal plane is postulated to form at the orifice.

The equation governing the motion of the bubble can be given from a balance between the upward mass acceleration of the fluid surrounding the bubble and the sum of the buoyancy force and the momentum force. Thus, the equation of upward motion is

$$
V_{B} \rho_{l} g+M_{g}=\frac{d}{d t}\left(\frac{11}{16} V_{B} \rho_{l} \frac{d s}{d t}\right)
$$
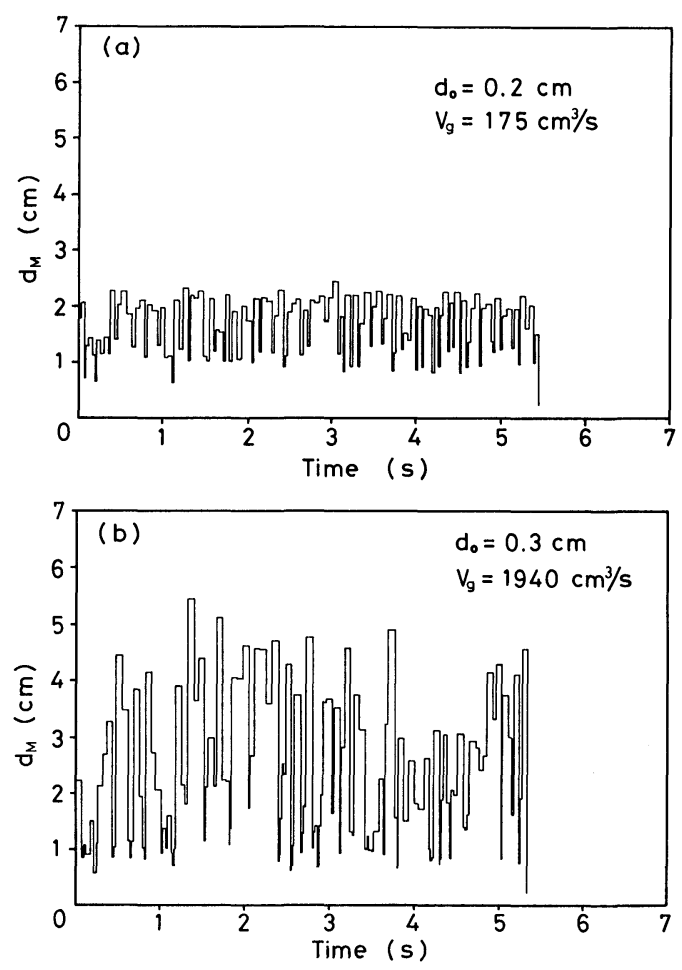

Fig. 4. Example of time change of $d_{M}$.

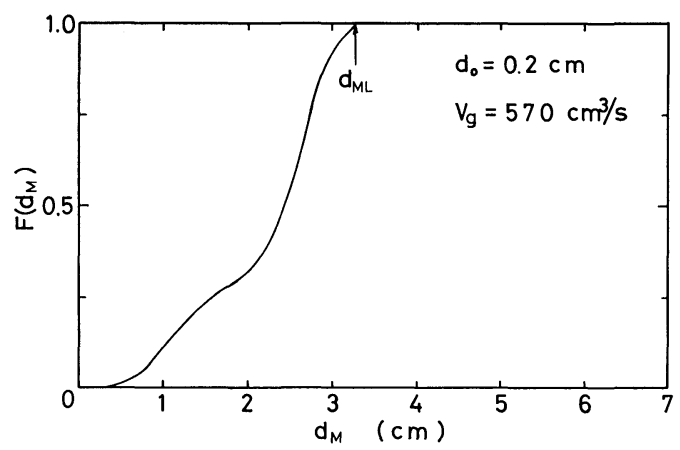

Fig. 5. Simplified cumulative distribution function of $d_{M}$.

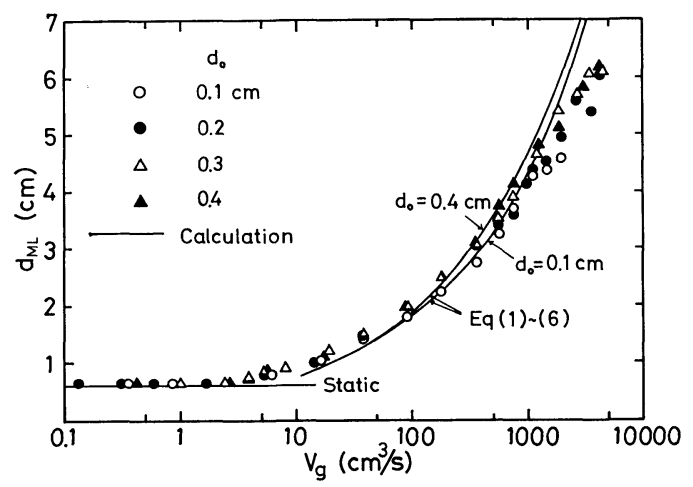

Fig. 6. Largest base diameter of bubbles plotted against gas-flow rate.

where, $V_{B}$ : bubble volume at time $t$

$\rho_{l}:$ liquid density

* Formation time of a bubble is defined as the time from the beginning of bubble formation to detachment.

** In Fig. 6 data in the sonic flow region are included.

*** See Fig. 7 . 
$g:$ acceleration due to gravity

$s$ : distance of center of bubble from orifice exit.

The virtual volume of the liquid surrounding the bubble is represented in Eq. (1) by $(11 / 16) V_{B}{ }^{7)}$ The force due to the rate of change of gas momentum is $M_{g}$, which is given by the following equations* for the two gas flows.

For the subsonic gas flow

$$
M_{g}=\frac{\kappa}{\kappa-1}\left(\sqrt{1+2(\kappa-1) M^{\prime 2}}-1\right) A_{o} P_{s}
$$

For the sonic gas flow

$$
M_{g}=\left(\sqrt{2(\kappa+1)} M^{\prime}-1\right) A_{o} P_{s}
$$

where, $\kappa: \quad$ specific heat ratio

$A_{o}$ : cross-sectional area of orifice

$P_{s}:$ hydrostatic pressure at orifice exit

$M^{\prime}$ : nominal Mach number.

The nominal Mach number is given by

$$
M^{\prime}=\frac{u_{o}}{a}=\frac{V_{g}}{a A_{o}}
$$

where, $u_{0}$ : nominal gas-flow velocity

$a$ : sonic velocity at room temperature.

With a constant gas-flow rate $\left(V_{g}\right), V_{B}=V_{g} t$. If $r$ is the radius of the bubble and $x$ is its base radius,

$$
\begin{aligned}
V_{B} & =\frac{1}{3} \pi(r+s)^{2}(2 r-s) \\
r^{2} & =s^{2}+x^{2}
\end{aligned}
$$

Under the condition that $s=0$ and $d s / d t=0$ at $t=0$, combination of Eqs. (1) (6) gives the relation between the base diameter of the bubble $(2 x)$ and the gas-flow rate $\left(V_{g}\right)$. This relation is shown in Fig. 6 by solid lines. Considering the assumption used in the calculation, the agreement between calculation and experiment is reasonable.

From the agreement between theory and experiment, the point source model may be considered to offer a good approximation of the mechanism of bubble formation under the present experimental conditions. Moreover, the assumption that bubbles of the largest base diameter are almost unaffected by the circulation of the liquid is proved to be reasonable except for the range of very high gas-flow rates.

\section{Average Diameter of Bubbles}

In Fig. 7 the average bubble diameter determined by using a resistance-probe technique for the bubbling regime is plotted against the gas-flow rate.** The general feature of the relationship shown in Fig. 7 is very similar to that for the base diameter of bubbles (Fig. 6). In Fig. 8 the bubble diameter is plotted against orifice Reynolds number for the three dimensions of orifices. Here, the data are compared with

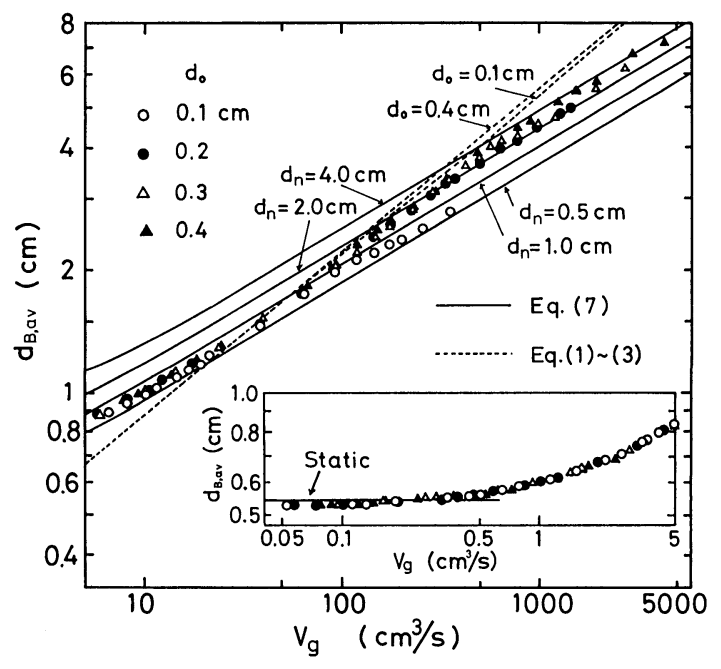

Fig. 7. Average bubble diameter plotted against gas-flow rate.

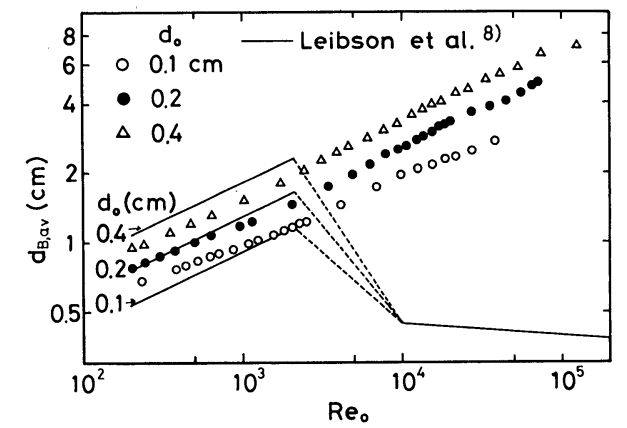

Fig. 8. Comparison of experimental data with correlation of Leibson et al.

the correlations obtained by Leibson et al. for the airwater system. ${ }^{8)}$

It seems that the bubble size obtained in the present study is comparable with the data of Leibson $e t$ $a l$. for the laminar-flow regime. However, this is only superficial. In the case of the study of Leibson et al. the gas chamber volume was so large as to produce an effect on the size of bubbles, while the gas chamber volume in the present experiment was negligibly small.

There is a large difference between the two data in the turbulent gas flow range. The bubble size obtained by Leibson $e t a l_{\text {。 }}$ for $\left(R e_{0}>2100\right)$ is concerned with fine bubbles produced from the break-up of larger bubbles formed at the orifice, while the bubble diameter measured in this study corresponds to large bubbles formed at the orifice

The diameter of bubble for the static formation is calculated in a manner similar to that of the maximum base diameter. As seen from the figure in the lower part of Fig. 7, good agreement is obtained between calculation and experiment. The calculation described below is for the bubble diameter at higher

\footnotetext{
* Derivation of Eqs. (2) and (3) is given in Appendix.

** Figure 7 does not include data at gas-flow rates higher than the critical rate described later, where the bubble diameter was unable to be determined accurately from the gas-flow rate and the bubble frequency.
} 
gas-flow rates.

On the basis of the assumption that at detachment the bubble radius $(r)$ is equal to the distance of the bubble center from the orifice exit $(s)$, the diameter of the detached bubble can be calculated from Eqs. (1) (3). In Fig. 7 the values of bubble diameter calculated for the orifice diameters of 0.1 and $0.4 \mathrm{~cm}$ are shown by dotted lines. Rough agreement is seen between calculation and experiment.

According to a previous study9) in the authors' Laboratory, the diameter of bubbles formed at the nozzle could be given by the following empirical equation:

$$
d_{B}=\left[\left(\frac{6 \sigma d_{n}}{\rho_{l} g}\right)^{2}+\left\{0.54\left(V_{g} d_{n}^{0.5}\right)^{0.289}\right\}^{6}\right]^{1 / 6}
$$

where, $\sigma$ : surface tension.

For nonwetted nozzles, the outer nozzle diameter is taken as the correlating nozzle diameter $\left(d_{n}\right)$. The orifice in the present case is missing the diameter corresponding to the nozzle outer diameter. Tentatively, giving $0.5,1.0,2.0$ and $4.0 \mathrm{~cm}$ for $d_{n}$, one calculates the bubble diameters as shown in Fig. 7 by solid lines. The increase in the value of $d_{n}$ with increasing the gas-flow rate brings about agreement of the bubble diameter between experiment and calculation.

\section{Jetting at High Gas-flow Rates}

\section{Bubbling and Jetting}

Figure 9 shows an example of the change of base diameter with time at high gas-flow rates. As seen from the figure, an apparent coincidence between the base diameter $\left(d_{M}\right)$ and the orifice diameter $\left(d_{o}\right)$ occurs over various time ranges. It is presumed from high speed motion pictures that in these time ranges the injected gas leaves the orifice as a continuous jet of gas. This phenomenon is called "jetting". According to Fig. 9(a), at the gas-flow rate of $1930 \mathrm{~cm}^{3}$ / $\mathrm{s}$ for the $0.2 \mathrm{~cm}$ orifice jetting is seemingly just initiated. As the gas-flow rate increases jetting occurs to a larger extent (Fig. 9(b)). The latter figure exhibits the alternation of jetting periods with bubbling periods.

In Fig. 10 distribution functions of $d_{M}$ are shown for almost the same flow rate for various orifice diameters. Jetting is observed to occur at 0.2 and $0.3 \mathrm{~cm}$ orifices. It is noticed that, irrespectively of large difference in the shapes of the curves of distribution function, the values of the largest base diameter of bubbles almost coincide for the three different orifice diameters.

The value of the distribution function, $F\left(d_{M}\right)$, at the bubble base diameter coinciding with the orifice diameter, as represented by the length of the vertical line, corresponds to time fraction for the occurrence of jetting (Fig. 10). One minus this value represents time fraction for bubbling.

In Fig. 11 time fractions for both bubbling and jetting are plotted against the gas-flow rate. As shown clearly in the figure, jetting is initiated at a critical gas-flow rate for each orifice diameter. With
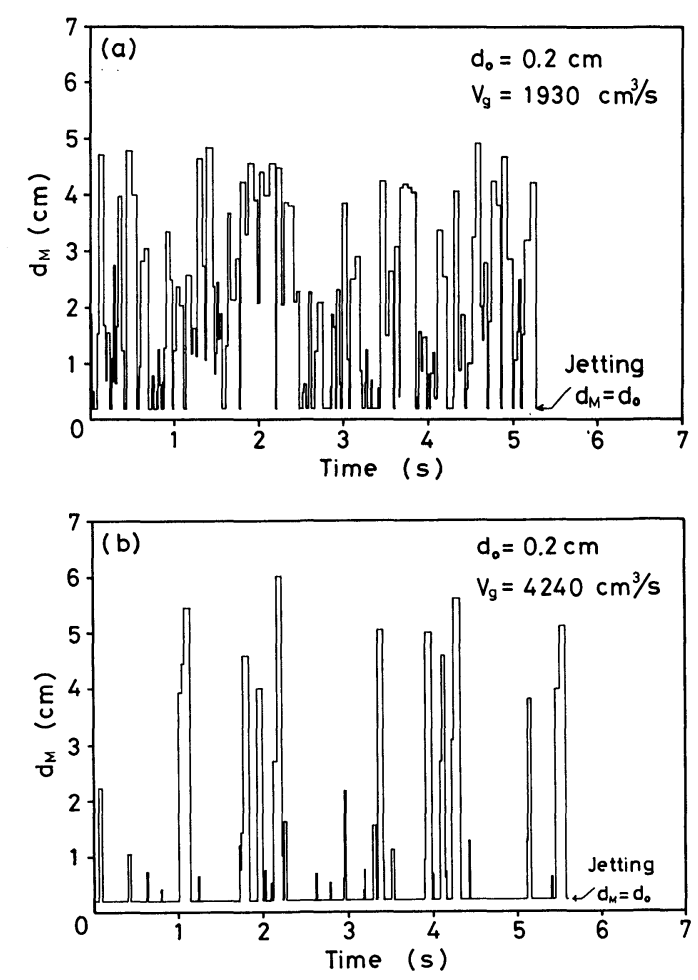

Fig. 9. Time change of $d_{M}$ in the range of transition from bubbling to jetting.

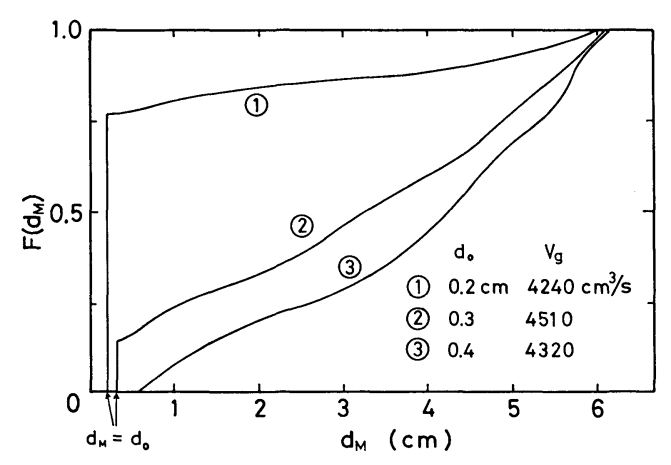

Fig. 10. Cumulative distribution function of $d_{M}$ for various injecting conditions.

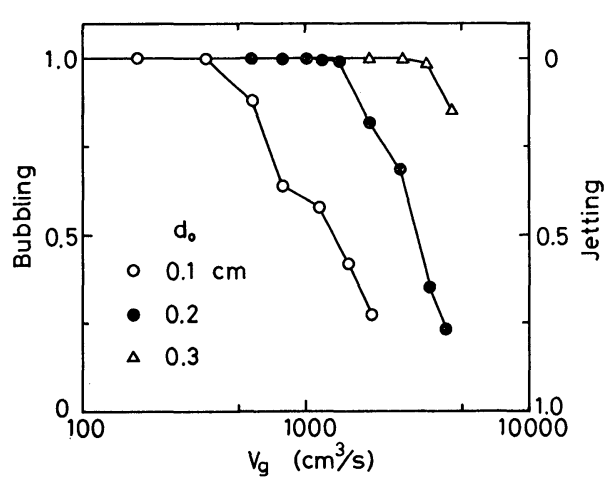

Fig. 11. Time fractions for bubbling and jetting plotted against gas-flow rate.

increasing gas-flow rate jetting fraction increases and bubbling fraction decreases. However, in the explored range of gas-flow rates perfect jetting did not 
occur. Thus, it has been cleared that a gradual change from bubbling to jetting takes place in a transitional gas-flow range.

In Fig. 12 bubbling-jetting time fractions are plotted for the three orifice diameters against the nominal Mach number $M^{\prime}$. As seen from the figure, the critical value of $M^{\prime}$ at which the transition from bubbling to jetting begins to occur is the same for the three diameters of orifice. It is found that the value of the critical gas-flow velocity is a little bit larger than but very close to the nominal sonic velocity $\left(M^{\prime}=1\right)$ irrespectively of the orifice diameter.

Since the orifice is of straight nozzle type, the gasflow velocity at the orifice is not allowed to have values higher than sonic velocity. The real gas-flow velocity $\left(q_{o}\right)$ together with the real gas pressure $\left(P_{o}\right)$ at the orifice exit is derived on the basis of fluid dynamic theory (Appendix).

In the subsonic region, $P_{o}$ is equal to the hydrostatic pressure $P_{s}$. In the sonic region

$$
\frac{P_{o}}{P_{s}}=\sqrt{\frac{2}{\kappa+1}} M^{\prime}
$$

The subsonic-sonic boundary can be given by

$$
M^{\prime}=\sqrt{\frac{\kappa+1}{2}}
$$

In Fig. 12 the subsonic-sonic boundary is indicated by a dotted line. The value of $M^{\prime}$ at this boundary is 1.1 for the present case of nitrogen injection.

As clearly shown in Fig. 12, jetting occurs in the sonic region. In this region, the gas pressure at the exit of the orifice is higher than the hydrostatic pressure and increases with increasing gas-flow rate (Eq. (8)). This high pressure of the injected gas overcomes the inertia force of the liquid surrounding the gas jet, leading to the occurrence of jetting. It is presumed that the gas jet penetrates into the liquid as a supersonic flow with shock waves. Details on this point will be examined in a subsequent paper.

\section{Comparison with Other Studies}

Several of previous investigators have directed their attention to an abrupt change in the jet behavior. ${ }^{10-12)}$ However, in the previous studies no pertinent definition of flow regimes has been given. Moreover, criteria for the difference in the flow regimes have not yet been established.

Ishibashi et al., ${ }^{10)}$ in the investigation of air jet behavior in water, have observed two different flow patterns very similar to those of bubbling and jetting observed in the present work. They used "bubble dispersion index" for the criterion of two different jet patterns. This is contrasted to the authors' finding that the bubbling-jetting transition occurs in the "sonic flow region".

Quite recently, Hoefele and Brimacombe ${ }^{12)}$ have investigated the behavior of gas jets in liquid by observing visually the gas injected into water, zincchloride solution, and a mercury bath. They distinguished two regimes of flow, bubbling and steady

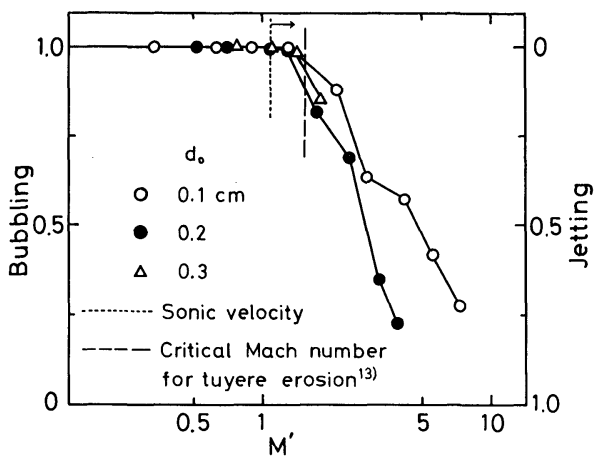

Fig. 12. Time fractions for bubbling and jetting plotted against nomimal Mach number.

Table 1. Comparison of flow condition determined for bubbling-jetting transition between the two

\begin{tabular}{|c|c|c|}
\hline & Present study & Hoefele \& Brimacombe ${ }^{12)}$ \\
\hline $\begin{array}{l}\text { Nominal } \\
\text { Mach } \\
\text { number }\end{array}$ & $M^{\prime}>1.3 \sim 1.4$ & $0.8<M^{\prime}<1.0$ \\
\hline $\begin{array}{l}\text { Modified } \\
\text { Froude } \\
\text { number }\end{array}$ & $\begin{array}{c}d_{0} \quad \text { Fr } \\
0.1 \mathrm{~cm}>2300 \sim 2800 \\
0.2>1100 \sim 1400 \\
0.3>750 \sim 950\end{array}$ & $\left.\begin{array}{c}d_{0} \\
0.2 \mathrm{~cm} \\
0.325 \\
0.476\end{array}\right\} 300<\mathrm{Fr}<1000$ \\
\hline Griterion & $M^{\prime}$ & $\mathrm{Fr}$ \\
\hline
\end{tabular}
studies.

jetting. The authors' definition for jet behavior in terms of bubbling and jetting has been given independently of the definition by Hoefele et al. Examining their paper, the authors find that the phenomena for bubbling and jetting implied by Hoefele et al. are different from those by the authors.

According to Hoefele and Brimacombe, the flow regimes depend on both modified Froude number Fr and the ratio of gas to liquid densities. As shown in Table 1, the values of Fr for the initiation of jetting observed in the present study vary with orifice diameter $d_{o}$. Namely, the bubbling-jetting transition in the present study is not delineated in terms of Fr.

Hoefele and Brimacombe seem to distinguish the two flow regimes from the point of continuity in the jet flow. In contrast to this, the authors have made the distinction by noting jet expansion at the orifice exit.

Sakaguchi et al. ${ }^{13)}$ investigated the erosion of a double-tuyere for injection of oxygen into molten steel. From their data giving the injection condition under which the tuyere refractory is little eroded, the authors have estimated the critical Mach number for erosion of the tuyere refractory used for injecting oxygen into steel bath. In Fig. 12 the value of this critical Mach number is shown by a dot-dash line. It is very interesting that the estimated value almost coincides with the critical velocity found in the present study. Thus, it has been cleared that the bubbling-jetting transition is of considerable significance in the practical problem of erosion of the tuyere refractory. 


\section{Summary}

A study has been made of the observation of behavior of gas jets injected into a mercury bath through an orifice at the vessel bottom. The results are summarized as follows:

(1) Two regimes of gas flow have been observed at the orifice exit: bubbling and jetting regimes. The latter regime is observed at high gas-flow rates.

(2) In the bubbling regime, the jet is shown to expand and form seemingly discrete bubbles. Except at very low flow rates, the largest base diameter of bubbles $\left(d_{M L}\right)$ is strongly dependent on the gas-flow rate, orifice diameter exerting only minor effect.

(3) The values of $d_{M L}$ could be reasonably explained by the point source model developed by Davidson and Schüler.

(4) Jetting is called for the phenomenon in which the injected gas leaves the orifice as a continuous jet of gas.

(5) The change from bubbling to jetting begins to occur at a critical gas-flow velocity which is a little bit larger than but very close to the flow velocity corresponding to the subsonic-sonic boundary irrespectively of the orifice diameter.

(6) The change is found to take place in a transitional gas-flow range. With increasing gas-flow rate jetting fraction increases and bubbling fraction decreases.

\section{Acknowledgements}

The authors wish to thank Professor M. Yasuhara at Department of Aeronautical Engineering, Nagoya University, for helpful discussions of fluid dynamic problems.

\section{REFERENCES}

1) M. Sano, Y. Fujita and K. Mori: Met. Trans., 7B (1976), 300.

2) M. Sano and K. Mori: Trans. JIM, 17 (1976), 344.

3) M. Sano and K. Mori: Trans. ISIJ, 20 (1980), 668.

4) M. Sano and K. Mori: Trans. ISIJ, 20 (1980), 675.

5) W. Fritz: Phys. Zeitsch., 36 (1935), 379.

6) G. Macdougall and C. Ockrent: Proc. Roy. Soc., A180 (1942), 151.

7) J. F. Davidson and B.O.G. Schüler: Trans. Inst. Chem. Eng., 38 (1960), 144 and 335.

8) I. Leibson, E. G. Holcomb, A. G. Cacoso and J. J. Jacmic: AIChE J., 2 (1956), 296.

9) K. Mori, M. Sano and T. Sato: Trans. ISIJ, 19 (1979), 553.

10) M. Ishibashi, K. Shiraishi, S. Yamamoto and M. Shimada: Tetsu-to-Hagané, 61 (1975), S111.

11) A. E. Wraith and M. E. Chalkley: Advances in Extractive Metallurgy, ed. by M. F. Jones, Inst. Min. Metal., London, (1977), 27.

12) E. O. Hoefele and J. K. Brimacombe: Met. Trans. B, 10B (1979), 631.

13) S. Sakaguchi, M. Ishibashi, M. Yoshii, S. Yamamoto and H. Kajioka: Tetsu-to-Hagané, 63 (1977), S534.

\section{Appendix}

Taking into consideration compressibility property of gas, one will derive equations relating to the gasflow through a submerged orifice.

\section{Basic Equations}

The volumetric gas-flow rate at the atmosphere pressure $P_{a t}$ and room temperature $T_{R}$, represented by $V_{g}^{\prime}$, may be calibrated for the effect of the hydrostatic pressure $P_{s}$ at the submerged orifice by

$$
V_{g}=V_{g}^{\prime}\left(P_{a l} / P_{s}\right)
$$

The nominal gas-flow velocity $u_{o}$ and the nominal Mach number $M^{\prime}$ are defined by the equations

$$
\begin{aligned}
& u_{o}=V_{g} / A_{o} \\
& M^{\prime}=u_{o} / a
\end{aligned}
$$

where $A_{o}$ is the cross-sectional area of the orifice exit and $a$ is the sonic velocity at room temperature.

Let $P_{o}, T_{o}$ and $q_{o}$ be the real gas pressure, gas temperature, and linear gas-flow velocity at the orifice exit. Then, from the gas law for the perfect gas,

$$
\frac{u_{o}}{q_{o}}=\frac{P_{o}}{P_{s}} \frac{T_{R}}{T_{o}}
$$

Suppose that a gas at $q_{o}=0$ and temperature $T_{m}$ changes adiabatically into gas flow at linear gas-flow velocity $q_{o}$ and temperature $\mathcal{T}_{o}$. Fluid dynamic theory gives the following equations.

$$
\begin{aligned}
& \frac{T_{m}}{T_{o}}=1+\frac{\kappa-1}{2} \frac{q_{o}^{2}}{a_{o}^{2}} \\
& \frac{T_{m}}{T_{o}}=\frac{a_{m}^{2}}{a_{o}^{2}} \ldots \ldots \ldots \ldots
\end{aligned}
$$

where $a_{m}$ and $a_{o}$ are the sonic velocity at temperature $T_{m}$ and $T_{o}$, respectively, $\kappa$ is the specific heat ratio $C_{P} / C_{v}$, and $T_{m}$ is the total temperature or stagnation temperature.

As an approximation, we assume that

$$
\begin{aligned}
T_{m} & =T_{R} \\
a_{m} & =a
\end{aligned}
$$

Then, Eqs. (A-4) to (A-6) become Eqs. (A-4') to $\left(\mathrm{A}-6^{\prime}\right)$.

$$
\begin{aligned}
& \frac{M^{\prime} a}{q_{o}}=\frac{P_{o}}{P_{s}} \frac{T_{R}}{T_{o}} \ldots . . \\
& \frac{T_{R}}{T_{o}}=1+\frac{\kappa-1}{2} \frac{q_{o}^{2}}{a_{o}^{2}} . \\
& T_{R}=\frac{a^{2}}{a_{o}^{2}} \ldots \ldots \ldots \ldots \ldots . . .
\end{aligned}
$$

Substituting Eqs. (A-4') and (A-6') into Eq. (A-5') yields

$$
\frac{\kappa-1}{2} \frac{q_{o}^{2}}{a^{2}}+\frac{1}{M^{\prime}} \frac{P_{o}}{P_{s}} \frac{q_{o}}{a}-1=0
$$

In the above formulation, it is assumed that friction loss in the orifice is negligibly small.

\section{Gas Pressure and Gas-flow Velocity}

Two cases of gas-flow are distinguished.

(1) Subsonic Gas-flow

In this case, the gas pressure at the orifice exit may be equal to the hydrostatic pressure as 


$$
P_{o}=P_{s}
$$

Substituting Eq. (A-9) into Eq. (A-5") gives

$$
\frac{q_{o}}{a}=\frac{-1+\sqrt{1+2(\kappa-1) M^{\prime 2}}}{(\kappa-1) M^{\prime}}
$$

for the real gas-flow velocity at the orifice exit.

(2) Sonic Gas-flow

For the orifice of straight nozzle type employed in the present study, the maximum velocity that can be reached is sonic velocity. That is, the gas-flow velocity at the orifice exit in the sonic gas-flow region is represented as

$$
q_{o}=a_{o}
$$

Applying Eq. (A-11) to Eqs. (A-4') to (A-6') leads to Eqs. (A-12) and (A-13) representing the gas pressure at the orifice exit and the real gas-flow velocity, respectively.

$$
\begin{aligned}
\frac{P_{o}}{P_{s}} & =\sqrt{\frac{2}{\kappa+1}} M^{\prime} \\
\frac{q_{o}}{a} & =\sqrt{\frac{2}{\kappa+1}} \cdots .
\end{aligned}
$$

As seen from Eqs. (A-12) and (A-13), the orifice gas pressure increases with increasing $M^{\prime}$, while the gas-flow velocity remains constant.

(3) Critical Condition

The critical gas-flow velocity expressed in terms of the nominal Mach number for the transition between subsonic and sonic velocity region can be obtained from substituting Eqs. (A-9) and (A-11) into Eqs. (A$\left.4^{\prime}\right)$ to $\left(A-6^{\prime}\right)$. Alternatively, putting Eq. (A-9) into Eq. (A-12) gives

$$
M^{\prime}=\sqrt{\frac{\kappa+1}{2}}
$$

for the critical condition. From this, it follows that: when $M^{\prime}<\sqrt{\frac{\kappa+1}{2}}$, the flow is subsonic. when $M^{\prime}>\sqrt{\frac{\kappa+1}{2}}$, the flow is sonic.

\section{Gas Momentum}

The gas momentum at the orifice exit $\left(M_{o}\right)$ and that of the bubble formed $\left(M_{B}\right)$ are represented by the equations

$$
\begin{gathered}
M_{o}=P_{o} A_{o}+\rho_{o} A_{o} q_{o}^{2} \\
M_{B}=P_{B} A_{B}+\rho_{B} A_{B} q_{B}^{2}
\end{gathered}
$$

The subscript $B$ in Eq. (A-16) refers to bubble.

The gas momentum $M_{g}$ which yields a force influencing the bubble formation is given by the difference between $M_{o}$ and $M_{B}$. Because of the difficulty in precisely formulating the two terms on the right-hand side of Eq. (A-16), an approximation is made as : $q_{B}=0$ and $A_{B}=A_{o}$. From this, it follows

$$
M_{g}=\left(P_{o}-P_{B}\right) A_{o}+\rho_{o} A_{o} q_{o}^{2}
$$

where $\rho_{o}$ is given by

$$
\rho_{o}=\frac{\kappa P_{o}}{a_{o}^{2}}
$$

If $P_{B}$ is assumed to be equal to the hydrostatic pressure at the location of the orifice exit $\left(P_{s}\right)$, one can write $M_{g}$ as shown below.

For the subsonic flow region, from Eqs. (A-9), (A10), (A-17) and (A-18)

$$
M_{g}=\rho_{o} A_{o} q_{o}^{2}=\frac{\kappa}{\kappa-1}\left(\sqrt{1+2(\kappa-1) M^{\prime 2}}-1\right) A_{o} P_{s}
$$

For the sonic flow region $\left(q_{o}=a_{o}\right)$, from Eqs. (A17) and (A-18)

$$
M_{g}=\left\{(1+\kappa) P_{o}-P_{s}\right\} A_{o}
$$

Combining Eq. (A-20) with Eq. (A-12) yields

$$
M_{g}=\left(\sqrt{2(1+\kappa)} M^{\prime}-1\right) A_{o} P_{s} .
$$

